Discourse in Filipino Philosophy

\title{
Recuerdos: Mga Pagbaliktanaw sa Lumahong Programang Pilosopiya ng Far Eastern University
}

\section{Christian Lemuel C. Afundar, Greg T. Dulay, and Elenita dLR. Garcia}

\begin{abstract}
Unknown to many Filipino teachers and students in Philosophy, Far Eastern University had its own bachelor's program in Philosophy. In fact, the Department of Philosophy of the university had some teachers and students who are considered as important Filipino philosophers, such as Benito Reyes, Florentino Timbreza, Antonio Cua, Romualdo Abulad, and Alfredo Co. Using the method of oral history, this paper attempts to reconstruct the story of the program and the Department of Philosophy of Far Eastern University. The researchers interviewed Florentino Timbreza as a former student, Zenaida Santiago-Martinez as a former college administrator, Clarita Villa-Alba as a former Philosophy teacher; and Alfredo Co as another former Philosophy teacher. This paper aims to find out why the bachelor's program in Philosophy was terminated; if it is appropriate to bring the program back; and if appropriate, how it should be designed to be more stable and sustainable. This paper can also be considered as a contribution to the broader project on the development of the history of Filipino philosophy.
\end{abstract}

Keywords: Far Eastern University, philosophy program, Filipino philosophy, oral history

\section{Introduksyon}

$\mathrm{L}$ ingid sa kaalaman ng marami ngayong Pilipinong guro at mag-aaral sa Pilosopiya, may panahon palang ang Far Eastern University (FEU) ay may sariling programang Batsilyer sa Pilosopiya. Ang dalawang mananaliksik sa proyektong ito na galing mismo sa nasabing pamantasan ay gulat na gulat nang marinig nila sa unang pagkakataon ang impormasyong

(C) 2018 Christian Lemuel C. Afundar, Greg T. Dulay, and Elenita dLR. Garcia https://www.kritike.org/journal/issue 22/afundar-dulay-garcia june2018.pdf ISSN 1908-7330 


\section{ANG LUMAHONG PROGRAMANG PILOSOPIYA NG FEU}

ito habang nasa Pambansang Kumperensiya ng Philosophical Association of the Philippines noong Abril 2017. Sa katunayan, mayroon ang Departamento ng Pilosopiya ng nasabing pamantasan na ilang guro at mag-aaral na maaaring ipagmayabang bilang mga mahalagang pilosopong Pilipino, tulad nina Benito Reyes, Florentino Timbreza, Antonio Cua, Romualdo Abulad, at Alfredo Co.

Humugot ng inspirasyon ang pananaliksik na ito sa tatlong institusyonal na pagsusuri na may kaugnayan sa pilosopiyang Pilipino na naisagawa na nina Preciosa Regina de Joya, tungkol sa mga pilosopong Pilipino ng Pamantasan ng Pilipinas, Pamantasang Ateneo de Manila (ADMU), Kolehiyong San Beda, at iba pa; ${ }^{1}$ F.P.A. Demeterio, tungkol sa mga pilosopong Pilipino ng Pamatasang De La Salle; ${ }^{2}$ at Emmanuel de Leon, ${ }^{3}$ tungkol sa mga pilosopong Pilipino ng Pamantasan ng Santo Tomas. Napagtanto ng mga mananaliksik na ang ganitong mga pag-aaral ay may malaking maiaambag sa pag-uunawa sa kasaysayan at kasalukuyang katayuan ng pilosopiyang Pilipino, pati na sa pilosopiya dito sa Pilipinas.

Gamit ang metodo ng oral history sinikap ng papel na ito na buuin muli ang kwento ng programang Pilosopiya ng FEU. Layunin ng papel na ito na makalikom ng ilang aral at kabatiran kung bakit naglaho ang programang Batsilyer sa Pilosopiya ng pamantasang ito; kung nararapat bang buhayin muli ang programa; at kapag nararapat man, kung paano ito ididisenyo para maging mas matatag at sustenableng programa. Maaaring ituring din ang papel na ito na isang ambag sa mas malawak na proyekto tungkol sa pagbubuo ng kasaysayan ng pilosopiyang Pilipino. Habang disertasyon at manuskrito ng aklat ang nagawa ng mga pagsusuri nina de Joya, Demeterio, at de Leon, isang maikling artikulo lamang ang nabuo ng kasalukuyang proyekto at itinuturing muna ito ng mga mananaliksik bilang panimulang pag-aaral sa kasaysayan ng pilosopiya ng FEU. Unang nakonseptuwalisa ang proyektong ito sa tulong at susog ng dalubhasa at historyador ng pilosopiyang Pilipino na si Demeterio.

\section{Metodolohiya}

Ang modernong oral history ay nagsimula sa pagkamulat ng Amerikanong dyurnalista at historyador na si Allan Nevins na madalas ang

\footnotetext{
1 Preciosa Regina de Joya, "In Search of Filipino Philosophy" (Ph.D. Dissertation, National University of Singapore, 2014).

${ }^{2}$ F.P.A. Demeterio, III, “The Intellectual Heritage of the Pioneering Lasallian Filipino Philosophers," (Unpublished Manuscript, 2015).

${ }^{3}$ Emmanuel de Leon De Leon, “Ang Intelektuwal na Pamana ng mga Pangunahing Tomasinong Pilosoper sa Kasaysayan ng Pamimilosopiyang Filipino: Quito, Mercado, Hornedo, Timbreza, Abulad, at Co" (Ph.D. Dissertation, University of Santo Tomas, 2017).

(c) 2018 Christian Lemuel C. Afundar, Greg T. Dulay, and Elenita dLR. Garcia https://www.kritike.org/journal/issue 22/afundar-dulay-garcia june2018.pdf ISSN 1908-7330
}

(c) BY-NC-ND 
mga nakasulat na teksto at rekord ay hindi sapat, o hindi mahagilap, para sa ilang historikal na pananaliksik. ${ }^{4}$ Nagsusulat si Nevins noong ng biograpiya ng Amerikanong Pangulong si Grover Cleveland na nabuhay noong uso na ang paggamit ng telepono at dumadalang na ang paggamit ng mga nakasulat na memorandum at sirkular. Kinapanayam ni Nevin ang mga nakatrabaho, kaibigan, at kamag-anak ng yumaong pangulo para mabuo niya ang ginagawang biograpiya. Ngunit may ginawang kakaiba si Nevins, inilagak niya sa isang arkibo ang mga rekord ng kanyang pakikipanayam para mapakinabangan pa ng mga dadating pang mananaliksik at historyador.

Ang oral history ay nakaugat sa pangangailangan ng isang historyador na makakuha ng impormasyon tungkol sa hindi pa masyadong naidokumentong penomenon o pangyayari. ${ }^{5}$ Humahanap ang oral na historyador ng kanyang mga impormante mula sa mga indIbidwal na may direktang kinalaman o karanasan sa paksa na kanyang sinasaliksik. Sistematikong kinapanayam ng historyador ang kanyang mga nahanap o napiling mga impormante. Planado dapat ang pagtatanong ng historyador para maihayag ng kanyang mga impormante ang kanyang kinakailangang mga impormasyon. Kaya hindi tamang paraan ng oral history ang basta hayaan na lamang ang impormanteng magsalita nang magsalita. ${ }^{6}$ Ang mga pakikipanayam ay dapat nakarekord, gamit ang audio o video na rekorder, at maaaring gawaan ng transkripsiyon. ${ }^{7}$ Ito ang dahilan kung bakit ang modernong oral history ay umusbong lamang matapos maimbento ang wire recording na maaaring bitbitin sa kinaroroonan ng mga impormante. ${ }^{8} \mathrm{Sa}$ puntong ito maaari nang magsulat ang historyador ng kanyang ulat o publikasyon.

Ang modernong oral history ay hindi kumpleto kapag wala ang proseso ng paglagak sa isang arkibo ng mga aktuwal na rekord at transkrip na nakalap mula sa proyekto. Ang mga nakalagak na rekord ay bukas dapat para sa mga darating na historyador na may kani-kanilang mga pananaliksik. Ang oral history ay tumutukoy sa sistematikong kinapanayam na mga salaysay mula sa respondents, tumutukoy rin ito sa mga rekord at transkrip, sa mga nakalagak na rekord at transkrip sa arkibo, pati na sa mga pag-aaral ng dadating na mga historyador na gagamit sa mga nakalagak na rekord at

\footnotetext{
4 Warren Nishimoto, "Introduction: Oral History in Educational Research," in Educational Perspectives, 40:2 (2007), 3.

${ }^{5}$ Valerie Raleigh Yow, Recording Oral History: A Guide for the Humanities and Social Sciences (Lanham, Maryland: Rowman \& Littlefield, 2015), 11.

${ }^{6}$ Michael V. Angrosino, Exploring Oral History: A Window on the Past (Long Grove, Illinois: Waveland Press, 2008), 6.

${ }^{7}$ Ibid.

${ }^{8}$ Yow, Recording Oral History, 2-3.
}

(c) 2018 Christian Lemuel C. Afundar, Greg T. Dulay, and Elenita dLR. Garcia https://www.kritike.org/journal/issue 22/afundar-dulay-garcia june2018.pdf

ISSN 1908-7330 


\section{ANG LUMAHONG PROGRAMANG PILOSOPIYA NG FEU}

transkrip, at pati na rin sa mga ulat at publikasyong nabuo kaugnay sa mga gawaing ito. ${ }^{9}$

Apat na lebel ng oral history ang sangkot sa proyektong ito. Una ay ang sistematikong pakikipanayam sa ilang piling impormante para makalap ang kani-kanilang salaysay tungkol sa programang Pilosopiya ng FEU. Kinapanayam ng mga mananaliksik sina Florentino Timbreza bilang dating mag-aaral, Zenaida Santiago-Martinez bilang dating administrador sa kolehiyong kinabibilangan ng nasabing programa, Clarita Villa-Alba bilang dating kasapi ng kaguruan ng Pilosopiya, at Co bilang isa pang kasapi ng kaguruan ng Pilosopiya. Nagkaroon muna ng mga inisyal na pakikipanayam ang mga mananaliksik kina Timbreza, Santiago-Martinez, at Villa-Alba batay sa pangkalahatan nilang masasabi tungkol sa naglahong programa ng Pilosopiya ng FEU. Matapos mapag-aralan ng mga mananaliksik ang mga inisyal na transkrip ay inilista na nila ang mga gabay na tanong para sa mga napiling impormante. Dito na idinagdag ang pangalan ni Co dahil lumabas sa inisyal na pakikipanayam na si Co pala ay nakapagturo rin sa nasabing pamantasan. Kahit hindi na niya naabutan ang programa ng Pilosopiya mismo, naabutan pa rin niya ang Departamento ng Pilosopiya at mayroon siyang alaala tungkol sa mga pangyayari at mga taong kasapi sa kwento ng Departmento ng Pilosopiya sa FEU. Ito rin ang naging gabay sa pagpili ng mga kapanayam.

Ang pangalawang lebel ng oral history na sangkot sa proyektong ito ay ang paglagay sa mga transkrip ng mga salaysay ng mga impormante sa papel na ito. Sa kawalan ng angkop na arkibong mapaglalagakan ng rekord at transkrip ng mga pakikipanayam, minabuti ng mga mananaliksik na sa binubuong artikulo na lamang ilathala ang nasabing mga transkrip. Sa pamamagitan ng isang publikasyon ay magagamit pa rin ang mga transkrip na ito ng sinumang dadating na mananaliksik na may interes sa paksang sinusuri ng proyektong ito. Ang pangatlong lebel ay ang pag-aaral sa mga nagawang transkrip para matugunan ang mga layunin ng proyekto na unawain ang paglaho ng nasabing programa, na malaman kung nararapat bang buhayin ang programa, at tiyakin na kung nararapat man itong buhayin muli, paano naman kaya ito ididisenyo para maging mas matatag at sustenable. Pang-apat ay ang artikulong ito na nabuo ng mga mananaliksik.

\section{Mga Alaala ng Isang Mag-aaral: Panayam kay Florentino Timbreza}

Ipinanganak si Timbreza sa Tayum, Abra. Siya ay nag-aral at nagtapos sa FEU ng kursong Batsilyer sa Pilosopiya noong 1962. Nagtapos siya ng kanyang Masterado sa Pilosopiya sa Ateneo de Manila University

${ }^{9}$ Ibid.

(c) 2018 Christian Lemuel C. Afundar, Greg T. Dulay, and Elenita dLR. Garcia https://www.kritike.org/journal/issue 22/afundar-dulay-garcia june2018.pdf ISSN 1908-7330

(c) $)$ BY-NC-ND 
(ADMU) noong 1971. Sa University of Santo Tomas (UST) naman siya nagaral at nagtapos ng kanyang Doktorado sa Pilosopiya noong 1980, kung saan isinulat niya ang kanyang disertasyon tungkol kay Lao Tzu. Ito ang kaunaunahang disertasyong pilosopikal dito sa bansa na isinulat at idinepensa sa wikang Filipino. Iginawad kay Timbreza ang pagiging full professor sa Trinity College, Quezon City kung saan siya ay naging Dean of Student Affairs. Nagturo siya sa De La Salle University (DLSU) noong 1983. Ginawaran siya ng DLSU ng parangal ng pagiging Lucian Athanasius Professorial Chair in Philosophy and Letters. Sa kasalukuyan, siya ang punong patnugot ng Filipino journal na Malay ng DLSU.

Unang nakapanayam ng mga mananaliksik si Timbreza noong ika17 ng Hulyo 2017 sa DLSU upang magkaroon sila ng preliminaryong impormasyon tungkol sa kanilang pag-aaral. Matapos mapag-aralan ng mga mananaliksik ang transkrip ng unang panayam, nagtala sila ng mga katanungang uungkat ng mas malalim sa karanasan ng kapanayam. Nagsagawa sila ng pangalawang panayam noong ika-15 ng Setyembre 2017 sa parehong lugar. Ang sumusunod ay ang transkrip ng pangalawang panayam.

Christian Lemuel C. Afundar (Kumakapanayam): Ano po ang dominanteng philosophical school na umiiral noon sa FEU?

Florentino Timbreza: Noong panahong iyon, laganap ang Scholastic, o masasabing neo-Scholastic dahil ang ilang propesor noon ay medyo liberal na rin. Hindi na lang basta Tomistikong sarado. Neo-Scholastic siguro ang masasabi ko. Magagaling naman sila. Meron ng oriental philosophy noon dahil nandoon si Dr. Benito Reyes. Iyon ang sa aking palagay ang umiiral na philosophical school of thought noong araw.

Greg Dulay (Kumakapanayam): Paano po kayo hinubog ng programang Pilosopiya ng FEU?

Timbreza: Dahil na rin sa aking mga magagaling na propesor, lalong lalo na kay Jose Espina. Siya ang masasabi kong humubog sa aking pag-iisip tungkol sa pagkakahilig ko sa pilosopiya. Magaling magturo. Hanggang ngayon iyong kanyang mga mannerism ay dala-dala ko pa rin sa pagtuturo. Kaya mahalaga iyong propesor o iyong guro mo sa pagtuturo, sapagkat malaking impluwensya sa pagkahubog ng iyong pag-iisip.

Afundar: Pati rin po ba sa pagsusulat ninyo, sila rin ba ang nag-encourage sa inyo?

(c) 2018 Christian Lemuel C. Afundar, Greg T. Dulay, and Elenita dLR. Garcia https://www.kritike.org/journal/issue 22/afundar-dulay-garcia june2018.pdf 


\section{ANG LUMAHONG PROGRAMANG PILOSOPIYA NG FEU}

Timbreza: Sa pagsusulat siguro, noong ako ay nagbibinata na sa amin, mahilig akong magsulat ng maiikling tula. Itong mga tulang ito ay aking hinahandog sa mga bagong kasal, halimbawa. Usong-uso sa Ilocos noon, sa Abra noon, iyong tinatawag nilang sarsuwela. Pagmagkaroon ng mga party, mga birthday party, o kaya kasalan, wala pa kasing ibang entertainment kundi sarsuwela. Wala pang radyo. Kaya kinakailangan kumanta ka o kaya they call that tula or daniw in Ilokano. Dumaniw ka ... parang balagtasan. Sa palagay ko doon nahubog ang interes ko sa pagsusulat.

Dulay: Maliban kay Dr. Jose Espina, sino pa po iyong mga naalala ninyo na naging propesor ninyo?

Timbreza: Si Lydia Almoro, magaling din iyon. Si Marcial Reyes. Meron pang certain Rivera. Hindi ko na nga lang maalala ang kanyang first name. Ethics yata ang itinuro niya noon, o kaya kung hindi ako nagkakamali parang tungkol sa Indian philosophy o Oriental philosophy. Marami rin ... nakalimutan ko na nga lang ang iba. Pero sila ang mga kaagad lulutang sa aking alaala. Iyon ang magigiting na mga propesor ko sa FEU noong araw.

Afundar: May ambag po ba ang pilosopikal na edukasyon ninyo mula sa FEU sa inyong pagiging isa ngayon sa mga pinakabatikang pilosopong Pilipino?

Timbreza: Maaaring biased ako dito sapagkat ang aking background talaga ay ang aking pag-aaral sa FEU. Oo naman, tulad ng sinabi ko, kung hindi dahil sa pagkakahubog ng aking pag-iisip, lalong-lalo na sa pilosopikal na pag-iisip sa FEU na dulot ng aking magagaling na propesor, palagay ko hindi ko narating ang aking naratnan kung anuman ngayon. Mahalaga ang background mo eh. Mahalaga iyong aking pilosopikal na background sa FEU.

Dulay: Ano po ang inyong masasabi sa paglaho sa programang Pilosopiya ng FEU?

Timbreza: Hindi ko matiyak pero marahil ... noong panahong iyon ang FEU ang may pinakamaraming mag-aaral. Mga 40,000 hanggang 42,000 ang kabuuang student population. Palagay ko, eventually siguro, ay pakaunti nang pakaunti na lang siguro ang nag-eenrol sa FEU. Kaya pakaunti nang pakaunti na rin siguro ang nag-me-medyor ng Philosophy. Maaaring iyong mga propesor ko ay nawala na rin siguro o kaya nag-transfer. Hindi ko alam kung nagretiro ba si Espina.

Dulay: Sa tingin ninyo po, malaki ba ang epekto sa isang pamantasan kapag nawala ang Philosophy program nito? 
Timbreza: Maaaring biased na naman siguro ako dito. Sa palagay ko may epekto, sapagkat tulad ng napag-aralan natin sa pilosopiya, ito talaga ang pundasyon ng paghahanap ng kabuluhan para malutas ang problema sa ating lipunan. Iyon din ang tinutukoy natin sa pilosopiya. Kung mawala talaga, sa palagay ko, maaaring magiging mahina ang epekto ng edukasyon. Sa bagay, meron naman yatang ethics o critical thinking pa rin sa FEU. Bukod pa siguro dito, kailangan pa ang malawakang paghahanap ng kabuluhan, at kinakailangan na ituro din ang iba pang sangay ng pilosopiya para lalo pang lumawak ang pag-iisip ng mga mag-aaral.

Afundar: Sa palagay po ninyo ay sapat ba ang graduate educational background ng mga propesor ninyo sa pilosopiya noon?

Timbreza: Dahil wala naman akong background talaga rin sa philosophy noon, iyong aking napag-aralan sa kanila ay sapat na para sa akin para mahubog ang aking pag-iisip at pagkakahilig sa pilosopiya. Pero noong ako ay nakapag-aral na sa graduate school, sa iba't ibang paaralan at eskwalahan, doon ko natuklasan na hindi pa pala sapat ang aking educational background. Pero mahalaga rin iyong aking educational background mula sa FEU. Parang stepping stone, wika nga, parang unang hakbang, dahil kung wala ang unang hakbang hindi ako nakatuntong sa iba pang pinag-aralan ko sa pilosopiya. Iyong background ko matatag, matibay. Iyon sa palagay ko ang kahalagahan ng aking pagmedyor sa Pilosopiya noong ako ay nasa FEU.

Dulay: Sa palagay po ninyo ay sapat ba ang mga ginagawang research ng mga propesor ninyo sa pilosopiya noon?

Timbreza: Kasi noong panahong iyon, hindi pa gaanong pinagdidiinan iyong research sa mga estudyante, kung hindi ako nagkakamali. Sapat na iyong, halimbawa, mga aklat na sinasabing basahin namin. Siyempre marami ring mga reference. Mayroon ding pagsa-submit ng mga term paper siguro. Pero baka hindi ito gaanong pinagdiinan noong panahon. Pero mayroon din. Parang isa sa mga requirement din iyong mga research paper o mga term paper na tinatawag.

Afundar: Kung sakaling maisipan ng administrasyon ng FEU na buuin muli ang nasabing programa, anong mga gabay at payo ang iyong maibibigay para sa administrasyon?

Timbreza: Siguro biased na naman ako kasi Philosophy ang medyor ko. Ang aking masasabi na lamang ... napakahalaga ng pilosopiya sa edukasyon.

(C) 2018 Christian Lemuel C. Afundar, Greg T. Dulay, and Elenita dLR. Garcia https://www.kritike.org/journal/issue 22/afundar-dulay-garcia june2018.pdf 


\section{ANG LUMAHONG PROGRAMANG PILOSOPIYA NG FEU}

Tulad ng ating mga dalubhasa, kahit itong ating mga bayani tulad ni Rizal, hindi mawawala iyong pilosopiya sa kanilang edukasyon. Napag-aralan nila ang pamimilosopiya, at ito ang dahilan kung bakit tumingkad ang kanilang pag-iisip. Kaya kinakailangan, sa palagay ko, na huwag kaligtaan ang pilosopiya bilang bahagi ng edukasyon. ... Ganoon din siguro ang masasabi ko sa mga guro tulad ninyo na nagtuturo ng pilosopiya. Ipagpatuloy ninyo, sapagkat ito ang interes natin. Walang ibang makakagawa nito eh. Huwag nating iasa sa iba. Kanya-kanya tayo ng interes, o kaya, o hilig, o special talent, o signature strength. Dapat nating palakasin ito, paunlarin ito, para naman makinabang ang iba, sapagkat ang katotohanan kung hindi ipapamahagi ay walang saysay. Parang pag-ibig, na magiging hindi tunay kapag ito ay hindi ibinibigay. Dito naman sa pilosopiya, ang katotohan ay walang kabuluhan kung hindi mo ito ipapamahagi sa iyong kapwa. Ito ang layunin ng pilosopiya, ang paghahanap natin ng katotohanan, pagtutukoy sa katotohanan. Ito ang ating ipapamahagi sa mga mag-aaral, upang mamulat rin sila sa katotohanang ating natuklasan. Malaking kontribusyon ito.

Dulay: Kahalintulad po ba ito sa sinabi ninyo dati na ang lahat na hindi ginagamit ay lumiliit?

Timbreza: Mayroon pang karugtong iyon ... kapag paliit nang paliit na ay hindi mo na talaga magagamit. Kaya sa akin, iyon ang kahalagahan ng pilosopiya sa aking buhay. Kung hindi dahil doon, maaaring hindi ako ang sinoman ako ngayon. Lalong-lalo na iyong pamimilosopiya sa wikang Filipino, mahalaga sa akin iyon, sapagkat malaki ang papel sa akin ang pagiging guro sa Filipino at ang pagtuklas at artikulasyon ng pilosopiyang Pilipino.

\section{Mga Alaala ng Isang Administrador: Panayam kay Zenaida Santiago-Martinez}

Si Santiago-Martinez ay ipininanganak sa lungsod ng Maynila. Nagtapos siya ng Bachelor of Arts in Speech and Drama sa FEU noong 1961, ng Bachelor of Science in Commerce sa parehong pamantasan noong 1992, at ng Master of Arts in Education at ng Doctor of Education mula sa pareho pa ring pamantasan noong 2003. Nagturo muna siya sa FEU High School ng tatlong dekada bago mag-umpisang maging guro sa antas tersiyaryo ng pareho pa ring pamantasan. Nanungkulan siya bilang tagapangulo ng Department of Communication mula 1997 hanggang 1994, at naging katuwang na dekano ng Institute of Arts and Sciences mula 1999 hanggang 2006. Nagretiro siya mula sa FEU noong 2010 at nagtuturo pa rin hanggang sa kasalukuyan sa Cainta Catholic College. 
Unang nakapanayam ng mga mananaliksik si Santiago-Martinez noong ika-30 ng Hulyo 2017 sa Cainta, Rizal. Matapos mapag-aralan ng mga mananaliksik ang transkrip ng unang panayam na nakatulong sa pagpili ng nararapat na mga katanungan sa pag-aaral na ito, nagsagawa sila ng pangalawang panayam noong ika-10 ng Setyembre 2017 sa Cainta, Rizal. Ang sumusunod ay ang transkrip ng pangalawang panayam.

Dulay: Bakit po naging pamilyar kayo sa kasaysayan ng programang Pilosopiya ng FEU?

Zenaida Santiago-Martinez: Kasi noon hindi pa ganoon karami ang mga department sa FEU. Well known din ang philosophy dahil parang isa ito sa mga original na programa ... philosophy at literature.

Dulay: Popular na po ba ang Philosophy Department noong mga panahon na iyon?

Santiago-Martinez: Yes. It was well known. Kasi mahusay na departamento iyon. Bakit mahusay? Because of the line up of faculty members and isa ito sa mga flagship course ng FEU. Si Marcial Reyes ay debonair ng philosophy. Si Benito Reyes, the front runner. Sina Lydia Almoro at Jose Espina pa. Si Timbreza ay produkto na nila iyon. Pero during his time, makikita mo na ang utak at potensiyal niya. Nakapaka-aktibo niya sa programa nila.

Dulay: Nabanggit ninyo po si Benito Reyes bilang front runner ng programa. Maaari po ba ninyo kaming kwentuhan tungkol sa kanya bilang propesor ng pilosopiya?

Santiago-Martinez: Naging propesor ko noon si Benito Reyes ng isang semestre lamang. Pero dahil kakaunti lang noon ang mga department at maliliit pa, so parang magkakakilala ang lahat sa loob ng FEU. Tapos, iyong mga anak niya ay naging kakilala ko, pati na ang misis niya. Kasi, si Mrs. Reyes also taught in FEU. Napakabait nitong si Benito Reyes. Kasi kung ano ang kailangan mo, bibigyan ka niya. Ang kanyang office ay parang open to all the students. Kung sino ang gustong magtanong, napaka-accommodating niya at generous.

Dulay: Kumusta naman po siya sa loob ng classroom?

Santiago-Martinez: Naging propesor ko siya sa isang subject. I think it was Literature. Kasi, he was into literature and philosophy. He was a deep thinker. Pero napaka... taong-tao ba. Napaka-humble. Hindi porke marunong siya ay

(C) 2018 Christian Lemuel C. Afundar, Greg T. Dulay, and Elenita dLR. Garcia https://www.kritike.org/journal/issue 22/afundar-dulay-garcia june2018.pdf

ISSN 1908-7330 


\section{ANG LUMAHONG PROGRAMANG PILOSOPIYA NG FEU}

alienated na sa kanya ang iba. Hindi siya ganoon. Pati mga anak niya hindi ganoon. Paglabas niya sa classroom, friend mo siya. Pero inside the classroom? It will be about the class that you should talk about. Marami siyang example, marami siyang activity for you to understand the lesson. Marami din siyang naisulat na libro. Marami siyang nai-contribute sa faculty journal. He was a prolific writer.

Dulay: Nabanggit ninyo po kanina si Marcial Reyes bilang debonair ng philosophy. Maaari po ba ninyo kaming kwentuhan tungkol sa kanya bilang propesor ng pilosopiya?

Santiago-Martinez: He was a philosophy professor. Guwapo at Spanish mestizo ito, balbas sarado. Ofelia Reyes ang pangalan nga kanyang asawa. Pero nagkahiwalay sila dahil mas bata si Marcial, guwapo, at kung tama ako may pagka-- flirt sa mga babae. Si Ofelia ay nag-aral sa Instituto de Mujeres. Kaklase siya ng mga kapatid ko. Hindi ko naging propesor si Marcial. Pero kilala ko siya because of Ofelia. Si Ofelia ay naging propesor ko. Then, later on, napabalita na naghiwalay sila. That was the time when Marcial left FEU.

Dulay: Nabanggit din po ninyo si Almoro kanina bilang isa sa mga pangunahing propesor ng Pilosopiya sa FEU. Maaari po ba ninyo kaming kwentuhan tungkol sa kanya?

Santiago-Martinez: Siya dati ay si Lydia Reyes. Naging propesor ko siya sa Philosophy. Late na siya nag-asawa. Pero noong nanganak naman ay halos dugtong-dugtong ang mga iyon. Pero napaka-solicitous na mother siya. Iyong mga anak niya, makikita mo, dadating silang mag-anak, silang mag-asawa, kapit-kapit ang mga anak niya. Sa FEU sila nag-aalmusal, nagsusuot ng sapatos kasi marami sila. Lawyer ang asawa niya. Pero maagang namatay iyon.

Afundar: Kumusta naman po ang kanyang pagtuturo ng pilosopiya?

Santiago-Martinez: Very exacting, very bookish. Lahat sa kanya may mga rule. Mas naging misteryosa siya as she grew older. Sa Cubao ang bahay niyan eh. May kaya naman sila. Ang anak niya yata ay pito. Lahat ng mga iyon ay nakapagtapos nang libre sa FEU.

Afundar: Napabayaan po ba niya ang kanyang pagtuturo dahil sa dami ng kanyang anak?

Santiago-Martinez: Hindi naman. Malinaw siyang magturo. Kaya nga lang intimidated ang mga student niya kasi iyong mga standard niya napaka- 
demanding eh. Kung magsasalita siya, hindi siya bababa sa antas ng mga estudyante, sila ang dapat aabot sa antas niya. Sa kanya, the time is mine, the subject is mine. Parang ganoon ang dating niya sa akin. Pero kinikilala din naman niya kung sino ang mga puwede niyang ma-intimidate o hindi. Ako, hindi niya ako na-intimidate. Commanding ang presence niya sa whole university. Kasi marunong. Kilala siya ng lahat dahil leader at she graduated, I think, magna or summa. At meron siyang mga librong isinulat eh. Si Lydia, maraming small talk. Maraming kwento ito eh. But she keeps on researching and studying. She did not stop. She loves to read and write. Hanggang puwede siyang mag-stay sa FEU, siya ay mag-stay. Hangang noong malapit na siyang mamatay ay narito pa rin siya.

Dulay: Kahit nag-retire na po siya ay nagturo pa rin sa graduate school noon. Naabutan pa kasi namin siya.

Santiago-Martinez: Oo! Kasi nga gusto niya ang ginagawa niya eh. Noong araw, ang tawag namin sa kanya ay babaeng nagsusuot chandelier. Kasi iyong hikaw niya ay parang chandelier. Siya ay taong totoo sa kanyang sarili. It was like, I do not care what you say, basta ito ako. Ako ay ako, wala akong pakialam sa inyo. Ganoon siya. Hindi siya ruled by the society.

Dulay: Nabanggit din po ninyo si Espina kanina bilang isa sa mga pangunahing propesor ng pilosopiya sa FEU. Maaari po ba ninyo kaming kwentuhan tungkol sa kanya?

Santiago-Martinez: Si Espina naman at ang kanyang misis ay naging propesor ko silang pareho sa pilosopiya. Silang dalawa ay propesor ng pilosopiya sa FEU. Jose Espina also wrote philosophy. Bilang propesor at estudyante lamang ang pagkakakilala ko sa kanya. Wala kaming mga small talk. Kasi iyong mag-asawa na iyon ay dadating sa FEU, magtuturo, tapos aalis na. Philosophy subjects ang itinuturo ni Jose Espina. Kapag nagtuturo iyon nakapikit sa harapan ng klase. Nagsasalita iyon nang nakapikit. Compatible ang mag-asawang iyon, dahil sa realm ng kanilang thoughts they see one another. Malaking tao si Jose Espina. Parang dating nagpapari pero hindi natuloy. Hindi masyadong nagtagal sa FEU ang mag-asawang iyon. Mabait sila at hindi naninigaw. Ang pagtuturo nila ay hindi simplified. Malalim ang level ng pagtuturo nila. Jose Espina was very quiet and disciplined ang demeanor niya. You will respect him. Hindi ka magloloko sa kanya. Pero he did not talk that much. When he opened his mouth, it was all about the lesson. Wala itong small talk.

(c) 2018 Christian Lemuel C. Afundar, Greg T. Dulay, and Elenita dLR. Garcia https://www.kritike.org/journal/issue 22/afundar-dulay-garcia june2018.pdf 


\section{ANG LUMAHONG PROGRAMANG PILOSOPIYA NG FEU}

Dulay: Napag-alaman namin na mayroon palang produkto ang programa na naging batikang propesor ng Chinese philosophy sa Amerika. Si Antonio Cua po ba ay kilala ninyo?

Santiago-Martinez: Hindi ko masyadong nakasalamuha yan dahil nauna sa akin yan sa FEU at hindi naman siya nagturo doon.

Dulay: 1952 daw ito nagtapos ng Batsilyer sa Pilosopiya at Sikolohiya sa FEU. Siguro nga sa Amerika na ito nag-aral para sa mga gradwado niyang degri. Sino-sino pa po ba ang mga pinakabatikang propesor sa pilosopiya ng FEU?

Santiago-Martinez: Hindi naman sila marami eh. Parang sila iyong nabanggit ko ang talagang cream sa philosophy. Itanong mo kay Claire [VillaAlba] kung meron pang iba. Siguro mas bata na lang. Pero during their time, sila iyon. Hindi mo matatawaran ang kanilang ability at sila ay mga authors.

Dulay: Ano po ang inyong masasabi sa paglaho ng programang Pilosopiya ng FEU?

Santiago-Martinez: May mga science program na itinatag, kaya kumaunti ang mga nag-major sa Philosophy. It became a losing proposition for the university. Because if you have very few majors. ... You will have to have a full class kasi ang bayad sa mga propersor ay full lagi. So, losing proposition iyon. Hindi puwede na ganoon.

Dulay: Kung sakaling maisipan ng administrasyon ng FEU na buuin muli ang nasabing programa, ano pong mga gabay at payo ang inyong maibibigay para sa administrasyon?

Santiago-Martinez: Ang unang gawin dapat diyan, maski ano mang programa iyan, ay ang marketing. You market by way of publicizing those who were there before. Ito ang mga produkto namin. Ito ang mga nagtuturo sa amin. Gusto nating ma-regain iyong stronghold ng university. Philosophy should always be there.

Dulay: Sa programa ng Philosophy, ano po ba dapat ang kalidad ng isang guro para maging kwalipikadong magturo para sa nasabing programa?

Santiago-Martinez: Sa akin kasi, ang unang titingnan mo sa guro ay attitude. Kung willing ba ang guro, kung may passion ba siya. Kasi maski gaano karunong iyan kapag hindi siya committed, wala ring mangyayari. Hindi 
iyong nagtatanong lamang tungkol sa salary scale. Kahit na ang FEU ngayon ay very competitive.

Dulay: Sa tingin po ba ninyo, puwede at maganda bang isulong ang muling pagbuo ng programang Pilosopiya sa FEU?

Santiago-Martinez: Oo naman! Basta ang mga nandoon ay magtratrabaho. Maraming guro at propesor, pero dedicated ba sila sa trabaho nila? Ang mga faculty journals noon ay dominated ng mga propesor ng pilosopiya at literature. Talagang alam mo na high caliber.

\section{Mga Alaala ng Isang Guro: Panayam kay Clarita Villa-Alba}

Si Villa-Alba ay ipininanganak sa bayan ng Lucban, Quezon. Nagtapos siya ng Bachelor of Philosophy sa UST noong 1971. Kumuha siya ng Master of Arts in Letters sa FEU at natapos niya ito noong 2000. Nag-aral siya para sa degring Doktorado sa Panitakan sa UST. Nagturo siya ng pilosopiya sa FEU mula 1973 hanggang sa pagretiro niya noong 2015. Maliban sa pagiging guro sa pilosopiya sa FEU, naging coordinator din siya sa Logic at Ethics. Siya ay co-author ng librong Basic Logic at Righteous Living na ginamit sa FEU sa pagtuturo ng Logic at Ethics. Naging kasapi siya sa editorial board ng una at pangalawang librong pilosopiya ni Emerita Quito na nakasulat sa wikang Filipino.

Unang nakapanayam ng mga mananaliksik si Villa-Alba noong ika20 ng Agosto 2017 sa Sacred Heart Village, Quezon City. Tulad ng mga ibang panayam, matapos mapag-aralan ng mga mananaliksik ang transkrip ng unang panayam, nagsagawa sila ng pangalawang panayam sa mga katanungang nagbunga dito. Nakapanayam muli si Villa-Alba noong ika-3 ng Setyembre 2017 sa parehong lokasyon. Ang sumusunod ay ang transkrip ng pangalawang panayam.

Dulay: Bakit po naging pamilyar kayo sa kasaysayan ng programang Pilosopiya ng FEU?

Clarita Villa-Alba: Kasi, I belonged to the Philosophy Department from 1973 up to my retirement in 2015.

Dulay: Ano po sa palagay ninyo ang naging dahilan sa paglaho ng programang Pilosopiya ng FEU?

Villa-Alba: Sa palagay ko, ang unang dahilan ay bumaba ang mga enrollee. Puwede ninyong sabihin na bumagsak ang bilang ng mga enrollee sa

(C) 2018 Christian Lemuel C. Afundar, Greg T. Dulay, and Elenita dLR. Garcia https://www.kritike.org/journal/issue 22/afundar-dulay-garcia june2018.pdf 


\section{ANG LUMAHONG PROGRAMANG PILOSOPIYA NG FEU}

Philosophy program dahil sa panahong iyon ang mga course na inenrolan ng maraming estudyante na gustong makakuha ng mga lucrative job ay iyong Nursing, Medical Techonolgy, na doon naman talaga nag-e-excel ang FEU, at saka iyong Mass Communications at Psychology. The graduates of Nursing and Medical Technology of FEU and also of Medicine then, usually topped the board examinations. The enrollees in Mass Communications and Psychology were increasing. So, siguro naging unpopular ang Philosophy program.

Afundar: May iba pa ba po kayang dahilan bukod dito?

Villa-Alba: Jokingly, the late Dr. Lydia Almoro would say that Dr. Alfredo Co was one of the causes for the disappearance of the Philosophy program because of his strictness and rigor. Lahat naman ng nagtuturo ng philosophy ay mga Philosophy graduate. Alam naman natin na talagang mahirap ang mga subject at ang programa mismo. Tapos papahirapan pa ng mga propesor na sabihin nating talagang hindi lang istrikto kundi talagang nandoon para paiisipin ka nang todo-todo just like them. Kung paano sila mag-isip, gusto nila ganoon din mag-iisip ang mga estudyante.

Afundar: Ano po ang inyong masasabi sa paglaho sa programang Pilosopiya ng FEU?

Villa-Alba: Sayang din. Kasi meron din namang ibang mga pasulpot-sulpot na estudyante na nagtatanong kung bakit walang Philosophy program. Gusto sana nilang mag-major sa Philosophy. Kaso, wala na. Sana kung meron nga tayong graduates ng Philosophy, sila na lang ang magtuturo ng subjects sa Philosophy na natira. Usually, ang nagtatanong sa akin noon, mga mag-la-law. Interesado sila sa Philosophy as a pre-law program.

Dulay: Sa palagay ninyo po ba ay nagkaroon ng epekto ang pagkawala ng programa sa paraan ng pag-iisip ng mga mag-aaral sa pamantasan?

Villa-Alba: Hindi. I do not think napakalaki ang epekto. Noong mga 70s kasi, up to siguro mga 80s, ang mga estudyante ay masisipag pa mag-aral, mahuhusay pa sila noon. Lalo na at ang hawak ko ay mga Nursing student. Mahuhusay sila at masisipag mag-aral. So, dahil hindi naman ako aware agad noon na nawala na pala ang Philosophy program, para sa akin ay parang wala namang malaking epekto sa pag-iisip ng mga mag-aaral.

Afundar: Iyon pong mga estudyanteng nagtatanong sa inyo tungkol sa Philosophy program, ano kaya ang mga major nila? 
Villa-Alba: Political Science, usually. At saka yung mga may balak mag-aral ng law pagkatapos ng bachelor's degree.

Dulay: Sa tingin po ninyo bakit kaya sila nagkaroon ng interes sa Philosophy program?

Villa-Alba: Hindi naman nila nabanggit kumbakit. At saka, siyempre, halimbawa kami noon, noong nag-aaral pa ako, siyempre kapag sinabi mo na Philosophy major ka parang angat ka. Iba ang pakiramdam mo kaysa ito ang course niya, Psychology, ang course niya Political Science, tapos siya ay English major. Ganoon. Tapos, ikaw Philosophy. Parang iba ang tingin sa iyo. Iba ang regard sa iyo. Parang ang tingin sa iyo matalino ka talaga. . . astig. Ganoon. At saka there were those na nag-iisip na mas mahahasa ang kanilang reasoning kapag Philosophy ang course nila. Kaya, it will be a good preparation for a Law program.

Afundar: Kung sakaling maisipan ng administrasyon ng FEU na buuin muli ang nasabing programa, ano pong mga gabay at payo ang inyong maibibigay para sa administrasyon?

Villa-Alba: Naku, they should first conduct feasibility study kung gaano karami kaya ang estudyante na interesado. Kasi, kung kakaunti rin lang naman, I do not think na mabuksan nila ulit iyan, na mabubuo ulit iyan. Kung may possibility man na mabuo, first of all talaga dapat mga Philosophy graduate ang magtuturo.

Dulay: Hindi na po puwedeng maghigpit masyado ang mga propesor?

Villa-Alba: Depende yan eh. Dapat naman siguro mahigpit din ang mga propesor. Lalo na when it comes to assignments. Ibig kong sabihin, may ipapabasa ka halimbawa, tapos pupunta sa klase ang mga mag-aaral na hindi nagbabasa. Dapat kung may assignment, may assignment. Kung meron dapat i-report, i-report. Kung ipinabasa mo ang partikular na dialogue ni Plato, pagdating ng estudyante sa klase dapat nabasa nya iyon. Otherwise, propesor lang ang magsasalita nang magsasalita.

Dulay: Ano po ang puwede mai-suggest ninyo na magiging thrust ng Philosophy program na ito?

Villa-Alba: Dapat iyong may orientation sila sa lahat ng trend: Oriental, Western, Ancient, Medieval, at iba pa. Dapat alam nila ang mga ito. Siguro, later on, ang isang estudyante naman ay magkaroon siya ng partikular na

(C) 2018 Christian Lemuel C. Afundar, Greg T. Dulay, and Elenita dLR. Garcia https://www.kritike.org/journal/issue 22/afundar-dulay-garcia june2018.pdf

ISSN 1908-7330 


\section{ANG LUMAHONG PROGRAMANG PILOSOPIYA NG FEU}

kikilingan. Halimbawa, noong estudyante ako, gustong-gusto ko iyong Ancient Philosophy. Kahit na doon sa UST ay obligado kaming maging interesado sa Scholasticism at Thomism. Eh, okay lang iyon. Pero as a student, meron din tayong ayaw. Halimbawa, si Kant, si Hegel, mga Germans, ayaw ko sa mga iyon. Pero dahil estudyante ka ng philosophy, kailangan nating pagaralan kahit ayaw natin. Ngayon, kapag nagtuturo na tayo ng philosophy, kung ano ang paborito natin iyon ang mas matagal nating i-take up kaysa ibang topic. At saka mas malalim ang diskusyon natin sa mga iyong gusto natin.

Afundar: Ano po kaya ang puwedeng gawing pang engganyo sa mga estudyante para kumuha sila ng Philosophy bilang major, kagaya ng pagkakaengganyo ninyo sa pilosopiya noon?

Villa-Alba: Puwede nating sabihin na it is a very good preparation for all graduate courses. Ako, honestly, noong nag-umpisa ako sa kolehiyo, wala akong definite na course na gusto. Ang nangyari lang kasi, si Josephine Acosta-Dr. Josephine Acosta-Pasricha na ngayon-isa ito sa mga propesor ng philosophy sa UST pero pinsan siya ng nanay ko, ay nagsabi sa akin na kung wala pa akong definite na course eh mag-Philosophy na lang daw ako. So nag-enroll ako sa Philosophy. Eh nagustuhan ko naman, kaya tinapos ko na. I suppose na hindi naman ako nagsisisi na iyon ang kinuha ko, kasi nakapagtrabaho naman ako kaagad.

Dulay: Sa personal po ninyong pananaw, malaki ba ang naitulong sa inyo ng philosophy?

Villa-Alba: Oo. Malaki. Kasi, halimbawa, kapag nagtuturo ako ng literature, dahil philosophy ang background ko naging mas malalim ang aking analysis at reading sa mga literary work.

\section{Mga Alaala ng Isa Pang Guro: Panayam kay Alfredo Co}

Si Alfredo Co ay ipinanganak sa lungsod ng Maynila at nagbinata sa Bicol. Siya ay nag-aral at nagtapos sa UST ng kursong Batsilyer sa Pilosopiya noong 1972. Sa UST din siya nagtapos ng pag-aaral ng kanyang Masterado sa Pilosopiya noong 1974 at Doktorado sa Pilosopiya noong 1976. Si Co ay kumuha rin ng post-doktorado sa klasikal ng pilosopiyang Tsino sa Chinese University of Hongkong, at sa Sorbonne naman siya kumuha ng postdoktorado sa Comparative Philosophy kung saan ipinagkaloob sa kaniya ng gobyerno ng Pransya ang dalawang iskolarsyip. Si Co ay nagturo ng pilosopiya sa FEU (1973-1977), sa DLSU (1973-1989), at sa UST (1975) kung 
saan ginawaran siya ng titulong Professor Emeritus. Kasama ng ilang magaaral sa UST, itinatag ni Co ang Philosophy Circle of the Philippines (PCP) na naging inspirasyon ng pagkakatatag ng Philosophical Association of the Philippines (PAP), at ang Philosophical Association of the Visayas and Mindanao (PAVisMinda).

Naganap ang kaisa-isang panayam kay Co noong ika-23 ng Setyembre 2017 sa Araneta Subdivision, Quezon City. Ang sumusunod ay ang transkrip ng panayam na ito.

Dulay: During the 2017 National Conference of the Philosophical Association of the Philippines, you mentioned that there was a Philosophy program in FEU. That was the information that inspired us to do this project, as it motivated us to dig deeper into the reasons why it disappeared.

Alfredo Co: Mabuti naman at nagdulot pala ang aking presentasyon ng mabuting bunga.

Dulay: We have already interviewed Mrs. Villa-Alba and she believed that you are one of the witnesses of the closure of the Philosophy program.

Co: During our time with Clarita [Villa-Alba] there was no Philosophy program in FEU. There was ... but not during our time.

Dulay: But Mrs. Villa-Alba mentioned that during your time, the chairperson of the Philosophy Department was Dr. Jonas Zita.

Co: Yes. But there was no program in Philosophy anymore. The Department of Philosophy was just a servicing department for general education subjects.

Dulay: Noong panahon po na nandoon pa kayo sa FEU, ano po ang dominant philosophical school na umiiral?

Co: Wala. Kanya-kanya. Since most of the young people that came with me were trained by UST, like Alba, Zita, and Almoro, you could imagine that it was still Scholastic. The Oriental philosophy was not available because there was no course offering for it during that time. That was why if I handled Ethics, I did not teach Christian ethics only, but also Eastern ethical thoughts. That was not enough to make an impact for Eastern philosophy. If I handled Logic, walang lumalabas ... Hindi mo nga matatapos ang lesson mo sa Logic, papasukan mo pa iyon ng mas malawak na Oriental thinking? Of course, Lydia [Almoro] was also using Oriental frameworks in her Ethics class. So that was about it.

(c) 2018 Christian Lemuel C. Afundar, Greg T. Dulay, and Elenita dLR. Garcia https://www.kritike.org/journal/issue 22/afundar-dulay-garcia june2018.pdf

ISSN 1908-7330 


\section{ANG LUMAHONG PROGRAMANG PILOSOPIYA NG FEU}

Dulay: Ang pagtuturo po ba ninyo sa FEU ay nagkaroon ng bakas o kontribusyon sa inyong pagiging isa ngayon sa mga pinakabatikang pilosopong Pilipino?

Co: That is not answerable, because I was not there as a regular faculty member handling major Philosophy subjects. Probably, hindi nilinaw ni Clarita na we both belonged to a servicing department.

Afundar: Si Benito Reyes po ay kilalang Orientalista ng FEU. Siya ba ay nakasalamuha ninyo? May naiambag po ba siya sa pagiging Sinolohista ninyo?

Co: Hindi ko siya nakasalamuha. Mas nauna siya sa akin. I did not have the opportunity to meet him. He is much older than I. If Emerita Quito was 30 years older than I, I imagined that Reyes was probably 25 or 28 years older than I. The only way for you to know more about Reyes is to check his biography at Pamantasan ng Lungsod ng Maynila where he served as president. Kaya wala siyang naiambag sa akin. Ang pagkakakilala ko kay Reyes came very much later. Noong nagre-research na ako about Filipino philosophy, saka ko lang napuna na meron palang Oriental philosophy noon sa FEU. Formed na ako noon. Ang formation ko sa Chinese philosophy ay mula sa Chinese University of Hong Kong.

Afundar: Ano pa po ang nalalaman ninyo tungkol kay Benito Reyes?

Co: In the early 1950s Benito Reyes and Ricardo Pascual went to study in the United States. Emerita Quito, in 1961, went to study in Switzerland. Benito Reyes was the first man I mentioned. I stated that FEU had an eminent Philosophy teacher, Benito Reyes. Students would listen to his lectures on Indian philosophy. The novelty of Indian philosophy attracted his students and he became the earliest Filipino to have written a book on Eastern philosophy. The book is entitled Moments without Self. That book also made him the first Filipino expert in Indian philosophy. The students enthusiastically followed his lectures in FEU. FEU, however, failed to create a competent group of faculty members who could carry that philosophical tradition in that university. The philosophical climate in FEU died when he left.

Afundar: Bakit po sa tatlong pantas ay si Reyes ang una ninyong binganggit?

Co: Maybe I was referring to him that way because, indeed, he was the center of it all. It was the time when very few people really can come with confidence to lecture about anything with a very big impression, carrying a load of knowledge from abroad. 
Afundar: Paano po kaya nabuo ang interes ni Reyes para sa Eastern philosophy?

Co: Actually, he was adopted by an American couple who was into the Theosophical Society. He was brought to the United States and was introduced to theosophical learning. Evidently, theosophical learning will bring you to some sort of Eastern thoughts. Because of that he became very passionate with Eastern philosophy.

Dulay: Mukhang si Antonio Cua na batikan ring Sinolohista ay hindi po ninyo naabutan dahil mas nauna pa itong mag-aral kay Dr. Timbreza sa FEU.

Afundar: Nabalitaan po namin na isa kayo sa mga kilabot noong propesor noon sa FEU at kinatatakutan ng maraming mag-aaral sa pilosopiya. Ano po ang inyong reaksiyon sa reputasyong ito?

Co: Hindi ko alam. Many people translate that if students are afraid of me, they run away from me. But I am treating everybody the same. Whether you are from FEU, DLSU, UST and ADMU, pareho lang turing ko sa kanila. Kahit magkano pa ang pasahod ng institusyon sa akin. Hindi ka puwedeng magyayabang sa akin dahil lang may mataas kang pasahod. Mag-aaral ka sa akin. Iyan ay napakahalaga sa akin, dahil ang turing ko sa kanilang lahat ay pare-pareho. Iyan ay aking dala-dala hangang ngayon. I think that is a big part of my pedagogical discipline. I'll tell you, many people who are afraid of me still cherish me, they still love me. They are afraid because they know they cannot just walk into my class unprepared, but at the same time they still love me.... They love me because I am strict, and they hate me of course. But they cannot accuse me of something else. Kaya ang pagtukoy ninyo na ako ay "kilabot" ay figuratibo. Medyo unfair sa akin iyon. Talagang si Lydia [Almoro]. Sumalangit nawa siya. But do not worry about it. I am too confident about me.

Afundar: Bukod po sa inyo, sino sino pa ang mga pinakabatikang dalubguro sa pilosopiya ng FEU?

Co: Dahil wala na ang Philosophy program noon, wala na ring lumalabas na talagang batikan. Dahil ang Pilosopiya noon ay puro general o minor subject na lamang. Nawala na kasi ang pagkakataon na malinang nang husto ang karunungan. Ang aking expertise sa Chinese philosophy ay unang naibahagi ko sa DLSU kaysa UST. Dahil ang Oriental philosophy sa UST noon ay hindi pa gaano nabigyan ng puwang. Sa DLSU, alam ni Quito ang aking expertise, kaya sabi niya sa akin "you come here, you will teach Chinese philosophy in DLSU."

(c) 2018 Christian Lemuel C. Afundar, Greg T. Dulay, and Elenita dLR. Garcia https://www.kritike.org/journal/issue 22/afundar-dulay-garcia june2018.pdf

ISSN 1908-7330 


\section{ANG LUMAHONG PROGRAMANG PILOSOPIYA NG FEU}

Afundar: Ano po masasabi nyo sa paglaho ng programang Pilosopiya sa FEU?

Co: Siguro ang pagkawala ni Benito Reyes, na isa sa mga naunang iskolar na nag-aral sa ibang bansa to broaden their philosophical horizons at bumalik para magturo. ... Siya rin ang kauna-unahang Filipino expert in Indian philosophy. Wala na sigurong nakakaungos sa kanya na maaring mamuno sa departamento na may M.A. o Ph.D. ... Parang isang katawan na nawalan ng ulo ang programang Pilosopiya sa FEU. Parang sa kanya lang umiikot ang mundo ng Philosophy sa FEU. Ganun talaga ang problema, kaya, I always see it that if you wanted a Philosophy program, you cannot create a program that is centered on you. You have to consider the faculty members who will be involved in creating the program, the faculty members who could handle the program. Malaking trabaho iyon. Like him, Pascual of the University of the Philippines was very charismatic, not only in Philosophy but also in Political Science. Kaya lang ang pagkakaiba ng dalawa, scholar si Reyes at nagsulat siya, si Pascual hindi masyado. Thus, Reyes was the first Filipino philosophy scholar who published. Kaya kapag may ganoong tao, parang wala nang susunod na iba. Parang doon na lang umiikot ang mundo ng Philosophy program sa FEU.

Afundar: Sa palagay ninyo, importante ba ang programang Pilosopiya sa isang institusyon?

Co: Dahil ako ang chair ng technical committee ng Philosophy sa Commission on Higher Education (CHED), siyempre ang aking sagot ay oo, napakaimportante nito. Alam ninyo matatanda na kami. Mabuti naman at isinusulat ninyo ang kasaysayan na kagaya nitong inyong ginagawa. May mga tao kasing kinakalimutan ang mga maliit na bakas na naiambag ng mga pilosopo at pilosopiya sa isang institusyon. Maybe, ganoon ako, kahit saan ako pumunta, nag-iiwan ako ng bakas na maaring magdulot ng isang kabutihan. At least, sa research ninyong ito, ikinukuwento ko na meron kayo noong Philosophy program sa FEU.

Afundar: Sa palagay po ninyo ay sapat ba ang mga ginawang research ng mga propesor sa pilosopiya noon sa FEU?

Co: Dahil wala na ngang departamento, kanya-kanya na lamang sulat at lathala. Kung sino na lamang ang gustong umangat. If you want your work to be published, you will do it on your own. 
Afundar: Kung sakaling maisipan ng administrasyon ng FEU na buuin muli ang nasabing programa anong gabay at payo ang iyong maibigay para sa administrasyon?

Co: Kailangan ninyong mag-submit ng proposal, and there are two ways. First, look at the program and standards that we have created in CHED and then you should be able to craft the program in such a way that it pushes the mission-vision of the department or university. Ano ba ang gusto ninyong gawin sa departamento o pamantasan? Bakit kailangan pang magkaroon ng isang Philosophy program? Papatunayan ninyo ito. Pagkatapos, dapat maipakita ninyo na mayroon kayong vision ... at diyan makikita kung ano talaga ang binabalak ninyo. Malakas ba ang programa sa, for example, Continental philosophy o sa Analytic philosophy, o sa Oriental philosophy, o Comparative philosophy. Dapat lalabas ito.

Afundar: Ano pa po ang dapat naming gawin bilang mga guro ng Pilosopiya sa FEU?

Co: Kailangan ninyo ng certain profile ng faculty members. Kasi ang deparment chair ay kailangang may qualification. Check the qualification of faculty members based on the standards of CHED. Check the program of Philosophy. Kailangan na tuparin ninyo iyon for basic formation. Pero kailangan din ninyo na magdagdag ng bagay na iniisip ninyong huhubog sa isang uri ng Pilosopiya na gusto ninyong i-develop para kilalaning tatak FEU. Halimbawa, UST, tatak Iskolastsismo; ADMU, tatak penomenolohiya; at DLSU, eklektisismo ayon sa mungkahi ni Quito.... You are not independent to just open a Philosophy program. The program should conform with the standards designed by CHED. Kailangan ang department chair at tatlo hanggang apat pang faculty member ay may Ph.D., habang ang iba ay may M.A. Then, you convince the CHED panel na mayroon kayong magtuturo sa programa. Patunayan ninyo na qualified sila. Masalimuot isipin, pero if you can make it, it will be an achievement.

\section{Paglalagom}

Sari-sari mang kwento at alaala ang naibahagi ng mga nakapanayam ng mga mananaliksik, tatlo sa kanila ay tila sumasang-ayon sa isa't-isa na ang paglaho ng programang Pilosopiya sa FEU ay dala ng pagbabago ng interes o pangangailangan ng mga mag-aaral sa pamantasan. Ayon kay Villa-Alba, naging popular ang mga programa sa Nursing, Medical Technology, Medicine, at Psychology. Si Santiago-Martinez naman ay naniniwala na ang pagtatatag ng mga programa sa Sciences ang naghila ng mga estudyante, kaya nawalan ng mga nag-enrol sa programa ng Pilosopiya. Binanggit din ni Timbreza ang pagkawala ng mga estudyante sa Pilosopiya. Mas madali nga namang makita

(C) 2018 Christian Lemuel C. Afundar, Greg T. Dulay, and Elenita dLR. Garcia https://www.kritike.org/journal/issue 22/afundar-dulay-garcia june2018.pdf

ISSN 1908-7330 


\section{ANG LUMAHONG PROGRAMANG PILOSOPIYA NG FEU}

ang pagiging praktikal ng pagkuha ng mga nasabing kurso kaysa sa Pilosopiya na ika nga ay "walang maidedeposito sa bangko." Maaaring ang pag-uso ng pangingibang bansa sa mga nakaraang dekada ay nagtulak sa mga mag-aaral na kumuha ng degri sa mga disiplinang madaling makakuha ng gawain sa labas ng bansa. Dahil dito, mahirap nga namang panatilihin ang programang Pilosopiya kung hindi sapat ang mga estudyanteng nagbabayad ng tuition fees kung hindi na makabuo ang departamento noon ng isang klase, habang kailangang suwelduhan ng buo ang mga nagtuturo nito, tulad ng nailahad ni Santiago-Martinez.

Subalit nabanggit din ni Timbreza ang pagkawala ng mga gurong nagtuturo ng Pilosopiya sa FEU dahil sa paglipat nila sa ibang institusyon, pag-alis ng bansa, o pagreretiro. Nabanggit ng mga nakapanayam ang mga pangalan ng mga batikang kaguruan ng Pilosopiya sa FEU noon, tulad nina Benito Reyes, Marcial Reyes, Lydia Almoro, Jose Espina, at Antonio Cua. Malinaw na naipaliwanag din ni Co na ang departamento ng Pilosopiya ay tila "katawan ng nawalan ng ulo" nang nawala ang nagsimula nito, na si Dr. Benito Reyes. Tiniyak ni Co na ang buong programa ng Pilosopiya ay nakaikot lamang sa katauhan ni Reyes at wala nang nakaungos pa na maaaring mamuno ng departamento. Dahil hindi naibahagi ang kakayahang magpatuloy ng programa sa ibang tao, natural lamang na maglaho ito kasama ng pagkawala ng pasimuno nito.

Tungkol sa posibilidad na ibalik ang programa ng Pilosopiya sa FEU, lahat naman ng mga nakapanayam ay sumang-ayon na mabuting mangyari ito. Lahat sila ay tumukoy sa kahalagahan ng pilosopiya sa edukasyon. Ayon kay Villa-Alba, maraming mag-aaral ang nagtatanong tungkol sa programang Pilosopiya dahil ibig nilang kunin ito bilang pre-law course. Ayon din sa kanya, iba ang mag-major sa Pilosopiya. Ang tingin ng mga tao ay angat sa lahat ang kumukuha ng kursong iyon. Isa pa, magandang preparasyon din ito sa kahit na anong kursong pang-gradwado. Si SantiagoMartinez naman ay nagsabing maibabalik ang programang Pilosopiya kung ang magiging kaguruan nito ay magkakaroon ng dedikasyon sa trabaho. Naalala pa niya na ang mga lathalain noon sa FEU ay nadominahan ng mga propesor ng pilosopiya at literatura.

Si Timbreza naman, sa sadyang nagmamahal ng disiplinang Pilosopiya, lalo na ng pilosopiyang Pilipino, ay naghayag na ang pilosopiya ay mahalaga sa lahat ng tao dahil ito ang "pundasyon ng paghahanap ng kabuluhan sa buhay." Ipinahiwatig niya na malalim ang kahalagahan nito at hindi lamang para sa mga Philosophy majors, kung hindi sa lahat ng mag-aaral. Si Co naman na siyang namumuno sa technical committee ng Pilosopiya sa CHED ay natural na nagdiin sa kahalagahan ng pilosopiya. Dagdag niya rin, sa dahilang tumatanda na ang mga batikang guro sa Pilosopiya ay kailangang maipagpatuloy ng iba ang pagtuturo at pananaliksik at 
paglathala sa nasabing disiplina. Ito ay upang makapag-iwan ng bakas sa lahat na tatahak ng landas ng pilosopiya.

Kasama naman sa mga payo at mungkahi ng mga nakapanayam, nang tanungin sila kung paano sisimulan uli ang programa ng Pilosopiya, sakaling mapag-isipan ito ng FEU, ay ang magaling na marketing ng programa, at ang pagsasagawa muna ng isang feasibility study upang malaman talaga kung may interes nga sa programa at kung ito ay magiging sustenable. Para kay Santiago-Martinez, kailangang maisapubliko ang kadakilaan ng mga naging guro dito noon, pati na rin ang mga naging dakilang produkto ng programa upang maibalik sa FEU ang lakas ng pilosopiya. Kaya para sa kanya, ang kaguruang magtuturo nito ay dapat may katapatan sa gawain at may malasakit sa disiplina as sa mga mag-aaral nito. Idinagdag naman ni Timbreza na kailangang itaguyod ito bilang paraan ng pagbabago, pagbibigay ng kabuluhan sa buhay, at pamamahagi ng katotohanan.

Sina Villa-Alba at Co naman ay nagbigay-diin sa pangangailangang desisyonan ng FEU kung ano ang orientasyon ng programa ng Pilosopiya, kung ito ay maibabalik nga. Ito ba ay magiging Kontinental, Analitik, Oriental, Komparatibo, atpb. sa orientasyon? Ang payo pa ni Co ay tingnan muna ang pamantayan sa CHED, magkaroon ng mission-vision, itipon ang mga kinakailangan para maipagtanggol ng FEU kung bakit magtatayo ito muli ng programang Pilosopiya. Aniya, kailangang mapatunayan muna na may ganitong pangagailangan sa FEU. Masasabi rin, base sa nasabing obserbasyon ni Co ukol sa pagkawala ni Benito Reyes, na kailangang isang komunidad ang magsisikap sa ganitong proyekto upang hindi maging depende sa iisang tao ang programa. Kailangang may nakapilang tao na susunod sa pamunuan nito.

Ang apat na nakapanayam ng mga mananaliksik para sa pag-aaral na ito ay nakapagbigay ng maraming interesanteng impormasyon hindi lamang sa itinigil na programang Pilosopiya sa FEU. Nabalikan din nila sa kanilang mga alaala ang kultura sa nasabing pamantasan nang mga nakalipas na panahon. Nakagagalak marinig na sikat sa kampus ang mga guro sa Departamento ng Pilosopiya at mataas din ang tingin sa mga estudyante ng disiplina. Kaya nakalulungkot malaman na ang pinakamalaking dahilan ng paglaho ng programa ay ang pagliit ng enrolment dito. Mabuti na lamang at nagpatuloy ang pagtuturo ng pilosopiya sa FEU bilang ilang serviced general education courses, at naranasan pa ng mga magaaral ang pagtuturo ng ilang propesor ng pilosopiya tulad ni Dr. Alfredo Co.

Mahalaga rin ang mga naging deskripsyon ng mga nakapanayam tungkol sa katauhan ng mga namunong kaguruan sa programang Pilosopiya sa FEU. Matingkad silang nailarawan-lalo na sa salysay ni Dr. Zenaida Santiago-Martinez-bilang mahuhusay na guro at mababait na tao, na may

(C) 2018 Christian Lemuel C. Afundar, Greg T. Dulay, and Elenita dLR. Garcia https://www.kritike.org/journal/issue 22/afundar-dulay-garcia june2018.pdf 


\section{ANG LUMAHONG PROGRAMANG PILOSOPIYA NG FEU}

kaniya-kaniyang kakaibang ugali-ang ilan ay istrikto, ang ilan ay hindi, ngunit lahat ay seryoso sa klase at magaling sa pagtuturo. May mga naging pamilya sila na nakilala rin ng mga mag-aaral. Sa madaling salita, naipakita nila ang pagiging regular na tao sa lahat ng nakapagmasid kahit na itinuturing ang pilosopiya na kakaibang disiplina at ito ay siya namang nagaragdag sa paghanga sa kanila bilang mga alagad ng pilosopiya.

\section{Kongklusyon}

Sa mga panayam na ginawa, dalawa ang pumaibabaw na dahilan ng paglaho ng Departamento ng Pilosopiya sa FEU. Ang una ay ang pag-iba ng direksyon ng pamantasan, na tila sumunod sa mga nausong kurso tulad ng Nursing, Medicine, at Medical Technology. Mahirap mang tanggapin, malaki ang epekto ng mga salik na pang-pinansyal sa pagsustena ng mga kurso. Kung walang masyadong kumukuha sa Pilosopiya dahil nga sa tila wala itong praktikal na gamit, wala rin itong kita para sa pamantasan. Mahirap makipagsapalaran ang isang institusyon na panatilihin ang kurso na hindi na nakaka-ambag sa usaping pampondo. Ang masyadong maliit na enrolment sa Pilosopiya ay hindi kinayang panatilihin ang sariling departamento.

Ang pangalawa at kasing-halagang dahilan ay ang paglisan ng mga taong namuno o nagturo sa lumang departamento. Hindi madaling iwasan na iugnay ang tao sa institusyon, lalo na kung napakahusay niya at hindi siya makapagtakda ng kanyang mga kasunod. Nang mag-alisan ang mga gurong nagtaguyod ng Departamento ng Pilosopiya-lumisan man ng bansa o lumipat ng institusyon o tumigil sa pagtuturo, naging matamlay na rin ang departamento. Kung walang maipagmayabang itong guro, wala rin itong magagamit na "marketing material."

Ngunit ang pagkawala ng Departamento ng Pilosopiya sa FEU ay maaaring malaking kawalan sa pundasyon ng edukasyon. Ang pilosopiya ay ang naturingang puso ng humanidades. Kung wala itong sariling departamento at kung wala itong mga majors, malaki ang magiging puwang sa tinatawag na pangkalahatan o pangkabuoang (general o holistic) edukasyon. Ang pilosopiya ay itinuturing ding isa sa mga pinaka-buong paraan ng pag-iisip at pag-aaral, kung kaya ito ay isang mahusay na paghahanda para sa karagdagang pag-aaral tulad ng Bachelor of Laws. Kaya naman magiging mainam kung maibalik nga sa FEU ang programang Pilosopiya.

Ang pag-aaral na ito ay may kontribusyon rin sa larangan ng pilosopiya sa labas ng FEU. Nakadaragdag ito sa arkibo tungkol sa estado ng pilosopiya sa Pilipinas. Naging talaan din ito ng mga taong nagturo ng at nagsaliksik tungkol sa pilosopiya. Ang mga impormasyong naibahagi dito ay

makatutulong bumuo ng larawan ng kultura ng mga pantas pilosopo sa 
bansa. Ang mga ito ay makapagbibigay gabay sa mga nakababatang iskolar sa pilosopiya, anuman ang kanilang maging mithiin bilang mag-aaral, guro, o administrador sa akademiya o sa iba pang institusyon. Magagamit din itong datos para sa iba pang pananaliksik tungkol sa (h)istorya ng pilosopiya sa Pilipinas.

Mabuti sanang isipin na gugustuhin ng mga administrador, guro, at mag-aaral ng FEU na buhaying muli ang kanilang programang Pilosopiya. Gaya ng sinabi ng mga kapanayam, kung sakaling maisipan ng FEU na tahakin itong landas na ito, maraming kailangang gawin at ihanda. Mainam din na nariyan ang mga pamantayan sa CHED at pati na rin ang paggabay ng chairperson ng technical committee para sa Pilosopiya na si Co. Kung ito ay matutuloy, maganda ring mapag-aralan ang magiging proseso nito upang maihalintulad sa mga karanasan ng ibang mga pamantasan na nagtayo rin ng programang Pilosopiya.

Institute of Education, Far Eastern University, Philippines

Department of Literature and Humanities, Far Eastern University, Philippines

Department of Philosophy, De La Salle University, Philippines

\section{References}

Angrosino, Michael V., Exploring Oral History: A Window on the Past (Long Grove, Illinois: Waveland Press, 2008).

De Joya, Preciosa Regina, "In Search of Filipino Philosphy" (Ph.D. Dissertation, National University of Singapore, 2014).

De Leon, Emmanuel, "Ang Intelektuwal na Pamana ng mga Pangunahing Tomasinong Pilosoper sa Kasaysayan ng Pamimilosopiyang Filipino: Quito, Mercado, Hornedo, Timbreza, Abulad, at Co" (Ph.D. Dissertation, University of Santo Tomas, 2017).

Demeterio, F.P.A., III, “The Intellectual Heritage of the Pioneering Lasallian Filipino Philosophers," (Unpublished Manuscript, 2015).

Nishimoto, Warren, "Introduction: Oral History in Educational Research," in Educational Perspectives, 40:2 (2007), 3.

Yow, Valerie Raleigh, Recording Oral History: A Guide for the Humanities and Social Sciences (Lanham, Maryland: Rowman \& Littlefield, 2015).

(C) 2018 Christian Lemuel C. Afundar, Greg T. Dulay, and Elenita dLR. Garcia https://www.kritike.org/journal/issue 22/afundar-dulay-garcia june2018.pdf

ISSN 1908-7330 